\title{
SOLAR ARCADE FLARINGS AS MAGNETODYNAMIC PROCESS OF MASS AND ENERGY SHEDDING FROM ENERGIZED MAGNETIC REGIONS
}

\author{
Y. UCHIDA, S. HIROSE, S. CABLE, S. UEMURA, K. FUJISAKI, \\ M. TORII AND S. MORITA \\ Physics Department, Science University of Tokyo, Tokyo
}

\begin{abstract}
Prototypes of magnetic actions in producing and shedding the $\mathrm{X}$-rayemitting high temperature plasmas in various astrophysical objects are witnessed in the spatially-resolved form on the Sun by the Solar X-ray Satellite "Yohkoh". The most prominent of those are arcade flarings seen as powerful arcade flares in active regions with strong magnetic field. Larger scale fainter X-ray arcade formation observed at high latitudes, shedding a large amount of mass and energy as CME's (coronal mass ejections) also belongs to this category. Since some features found by the new observation by Yohkoh are incompatible with the so-called "classical model of arcade flarings", we advance an alternative model based on the quadruple magnetic sources in the photosphere.
\end{abstract}

\section{Introduction}

Solar flares are sudden releases of energy stored in the stressed magnetic field. A superhot plasma region is created due to the fast release of magnetic energy in the Alfven-transit time, much faster than the energy loss times by radiation or by heat conduction in the rarefied plasma in the corona. Coronal magetic field having a plasma frozen to it can store energy in the form of magnetic stress if it is distorted by the motion of the footpoints in the high $\beta\left(=p_{g a s} / p_{\text {mag }}\right)$ gas in the photosphere. If the magnetic field configuration has a neutral point (or an array of neutral points) in it, the stressed magnetic fluxes with the loaded mass and helicity can be transferred to other regions of lower stress, due to reconnections in the neutral point region. and can reduce the accumulated magnetic stress energy in the system. 


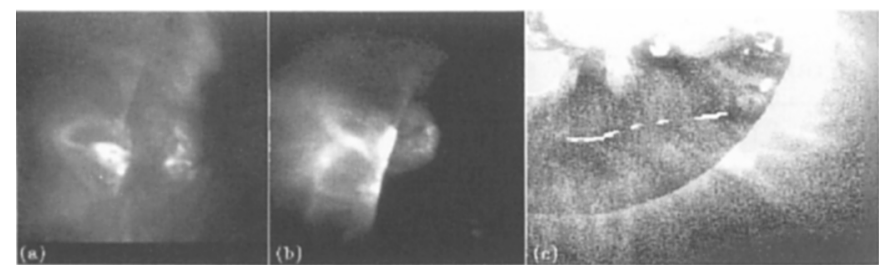

Figure 1. Quadrupolarity of Arcade Flares and Arcade Formations: High connections to both sides of the flare core and a brightening at the axis of the dark tunnel below in (a) Feb 21, 1992 flare, and in (b) Dec 2, 1991 flare. (c) Pre-event coronal structure on Jan 18, 1992, of the "Giant Cusp" event of Jan 25, 1992.

\section{Pre-event Corona around the Site of Arcade Flarings}

Recent observations by Yohkoh have revealed essential new information about the arcade flaring processes (Tsuneta et al. 1992). Detailed analyses of the observations of the still faint pre-event coronal structures of arcade flarings have revealed that there exist in the fainter pre-event corona, structures like the high connections from the top of the flare core back to the photosphere on both sides, or like the brightening at the axis of the dark tunnel below the cusped arcade (Uchida 1996), or the "overlapped dual arcades" in the case of high latitude arcade formation events (Uchida et al. 1997)(Fig 1). Those strongly suggest that the bipolar source picture that the "classical model" was based upon may not be valid. Corresponding to the "overlapped dual arcades", it was found that the photospheric magnetic field below them is not a simple bipolar region, but many patches of wrong polarities exist (cf., Martin 1990) on the other side of the previously called "polarity-reversal line", and the situation may correspond to quadruple arrays of magnetic sources, explaining the "overlapped dual arcades" as its separatric surface (Uchida 1997).

Also, the findings about the process of X-ray arcade formation itself, like the "spine" rising from below the X-ray arcade sometime after the dark filament rose, forming ultimately a feature like the "Giant Cusp" (Fujisaki et al. 1997), are also something not expected in the "classical model".

The so-called "classical model" (Sturrock 1966, and others) has a scenario that the magnetic arcade connecting the bipolar regions supports the dark filament on the top part of it, and the arcade is opened up by the rise of the dark filament, and a magnetic neutral point is created between the legs of the pulled out arcade. The reclosing of the once opened-up arcade through magnetic reconnection is thought to provide the difference in the energies of the opened and reclosed fields to the flare. Uchida (1980) noted that this model has a difficulty in energy that the energy of the dark filament rise should then be larger than the flare energy itself. One should then explain the energy of the dark filament rise, instead, but that is never 


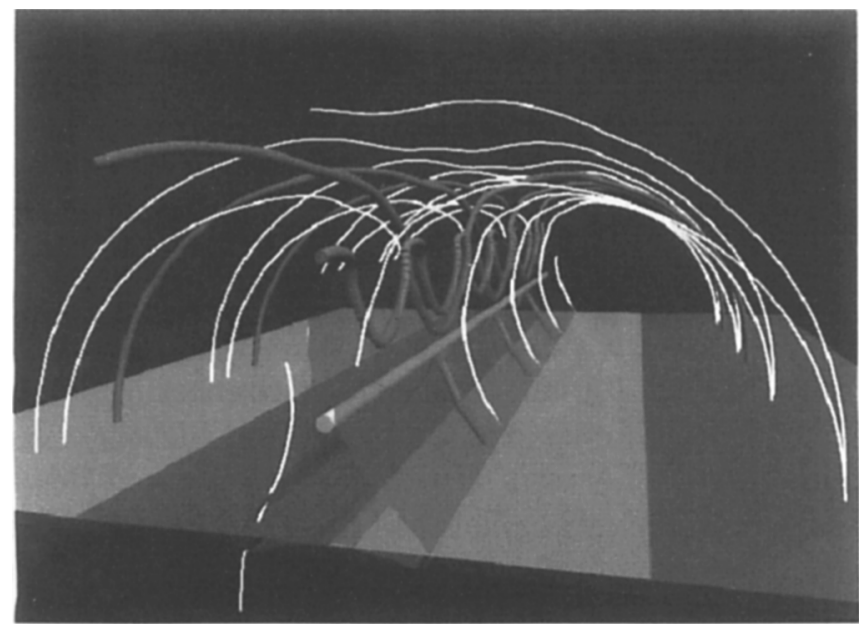

Figure 2. Magnetic Structure in the MHD Simulations of Hirose et al.(1998): The observed photospheric field with a large number of opposite-polarity patches on the other sides are modelized as quadruple arrays. The separatric surface in the field is the model's counterpart of the observed "overlapped dual arcades".

addressed. Also a question was raised about the suspension of the dark filament mass at the top of the otherwise convex arcade, especially initially when no dip has developed yet.

\section{The Process in the Quadruple Source Model}

Uchida et al. $(1980,1996,1997)$ advanced a model having quadruple magnetic sources in the photosphere, in place of the "classical model". In this model, there exist an array of neutral points in the field components $B_{\perp}$ (field perpendicular to the polarity-reversal line in the middle of the arrays of,,,+-+- sources) from the beginning. The longitudinal field $B_{\|}$dominating in this neutral sheet of $B_{\perp}$ suspends the dark filament gas in the form of a thin partition, held by the squeezed antiparallel part of $B_{\perp}$ above the central polarity reversal line (Fig 2). The gravity acting on the dark filament mass stabilizes this.

We have performed a 2.5D MHD simulations with the destabilization of the dark filament by a current injection from a small emerging magnetic region. We found in the simulations (Hirose et al. 1997) that the thin partition type dark filament can exist in equilibrium first, separating the oppositely-directed vertical $B_{\perp}$ on both sides. When the dark filament is squeezed out, the strong antiparallel vertical parts of $B_{\perp}$ pushed from both sides (the right and left quadrants) can start reconnecting in the part of the neutral sheet from which the dark filament has been squeezed out. A 
considerable amount of mass and helicity loaded on the reconnecting flux can be transferred directly across the separatric surface, as a part of the flux tube undergoes the reconnection at the center. The dark filament is dynamically passive here, and pushed up by the fluxes swelling up across the separatric surface, rather than actively pulling the arcade into a tipped structure as supposed in the "classical model".

The reconnection in this case produces the flare core, heated cuspshaped region, just like the observed cusps in arcade flares, but here, the heated part also has the upper connections connecting back the top of the heated cusp to the photosphere on both sides just as observed in Fig. 1(a),(b) (Uchida 1996). There is also a feature pressed down (S-shaped structure if seen from above), explaining the observed brightening feature near the axis of the dark tunnel. There are no counterparts for these in the "classical model".

The released part of the rising flux tubes will further be accelerated by the magnetic buoyancy, rolling down the slope of the magnetic potential gradient outwards (melon-seed effect). This final process in the mass and energy shedding, allowed by the occurrence of magnetic reconnection, may explain the large energy and mass in CME (coronal mass ejection)'s.

\section{Conclusion}

Observations by Yohkoh brought us some essential information about the global model of arcade flarings that was hidden in the still faint pre-event structures, supporting the quadruple magnetic source model, rather than the classical "opening up - reclosing" model.

These findings and new interpretations about energy and mass shedding from arcade flares and arcade formations will provide prototypes for their much greater versions in other active stars like dMe and RSCVn.

\section{References}

Fujisaki,K., et al. 1997, submitted to Publ.Astron.Soc.Japan.

Hirose, S., et al., 1997, in preparation.

Martin, S., in Dynamics of Quiescent Prominences, (Springer Lecture Note on Physics) 363, pp1-44.

Sturrock, P.A., 1966, Nature, 221, 695.

Tsuneta, S., et al., 1992, Publ. Astron. Soc. Japan, 44, L63-69.

Uchida, Y., 1980, in Skylab Workshop, Solar Flares, ed. P.A. Sturrock (University of Colorado Press), p67, and p110.

Uchida, Y., 1996, Adv. Space Res., 17, (4/5)19-28

Uchida,Y., et al., 1997, submitted to Publ.Astron.Soc.Japan. 